Original Research Article

\title{
Evaluation of cognition enhancing activity of pioglitazone in albino mice
}

\author{
Shruthi S. L. ${ }^{1}$, Kalabharathi H. L. ${ }^{2 *}$, Siddamma Amoghimath ${ }^{3}$
}

${ }^{1}$ Department of Pharmacology, SIMS, Shimoga, Karnataka, India

${ }^{2}$ Department of Pharmacology, JSS Medical College, Mysore, Karnataka, India

${ }^{3}$ Department of Pharmacology, GIMS, Gadag, Karnataka, India

Received: 08 October 2017

Accepted: 12 October 2017

*Correspondence to:

Dr. Kalabharathi H. L.,

Email: drshruthideepak@ yahoo.com

Copyright: (C) the author(s), publisher and licensee Medip Academy. This is an openaccess article distributed under the terms of the Creative Commons Attribution NonCommercial License, which permits unrestricted noncommercial use, distribution, and reproduction in any medium, provided the original work is properly cited.

\begin{abstract}
Background: Worldwide, 47.5 million people have dementia, with just over half $(58 \%)$ living in low- and middle-income countries. Every year, there are 7.7 million new cases. The estimated proportion of the general population aged 60 and over with dementia at a given time is between 5 to 8 per 100 people. The total number of people with dementia is projected to be 75.6 million in 2030 and almost triple by 2050 to 135.5 million.

Methods: All animals were allowed to acclimatize with laboratory conditions at least two weeks before starting the experiment and they were maintained under the same condition throughout the experiment. They were given food and water ad libitum. The experiments were performed as per the Committee for the Purpose of Control and Supervision on Experiments on Animals (CPCSEA) guidelines. The animals were subjected to experimentation between 0900-1600 hours in noise free atmosphere with ambient temperature $23-300^{\circ} \mathrm{C}$.

Results: The Pioglitazone treated groups showed statistically significant results in the SDL when compared to the control group ( $p<0.01$ on both days) and the scopolamine group ( $\mathrm{p}<0.01$ on both days) on both acquisition day and retention day.

Conclusions: There was no significant difference in SDL in standard and Pioglitazone treated groups on both acquisition day and retention day.
\end{abstract}

Keywords: Albino mice, Cognition, Pioglitazone

\section{INTRODUCTION}

Neurodegenerative disorders represent a significant healthcare issue with an increasing aging population. Neurodegeneration is an umbrella term for the progressive loss of structure or function of neurons, including death of neurons. Neurodegeneration can be found in many different levels of neuronal circuitry ranging from molecular to systemic. Some of the neurodegenerative disorders include:

- $\quad$ Alzheimer's disease (AD) and other dementias

- $\quad$ Parkinson's disease (PD) and PD-related disorders

- $\quad$ Prion disease

- $\quad$ Motor neuron diseases (MND)
- Huntington's Disease (HD)

- $\quad$ Spinocerebellar ataxia (SCA)

- Spinal muscular atrophy (SMA)

As research progresses, many similarities appear that relate these diseases to one another on a sub-cellular level. Discovering these similarities offers hope for therapeutic advances that could ameliorate many diseases simultaneously. There are many parallels between different neurodegenerative disorders including atypical protein assemblies as well as induced cell death. ${ }^{1,2}$

Dementias are responsible for the greatest burden of disease with Alzheimer's representing approximately 60$70 \%$ of cases. 
Alzheimer's disease (AD) was first described by Alois Alzheimer more than a century ago in Germany and it constitutes one of the most common causes of senile dementia.

Dementia rates are growing at alarming proportion in all regions of the world and are related to population aging. Neurologic conditions, including dementia, were estimated by the Global Burden of Disease 2010 Study as the third leading cause of years lived with disability at global level. ${ }^{3}$ Worldwide, 47.5 million people have dementia, with just over half $(58 \%)$ living in low- and middle-income countries. Every year, there are 7.7 million new cases. The estimated proportion of the general population aged 60 and over with dementia at a given time is between 5 to 8 per 100 people. The total number of people with dementia is projected to be 75.6 million in 2030 and almost triple by 2050 to 135.5 million. Much of this increase is attributable to the rising numbers of people with dementia living in low- and middle-income countries. ${ }^{4}$ The prevalence of dementia rapidly increases from about 2-3\% among those aged $70-75$ years to $20-25 \%$ among those aged 85 years or more. ${ }^{5}$ Over this age, there is paucity of data to affirm whether dementia prevalence keeps increasing or stabilizes. ${ }^{6}$

Alzheimer's disease (AD) and other forms of dementia are a growing public health problem among the elderly in developing countries, whose aging population is increasing rapidly. It is estimated that by the year 2020, approximately $70 \%$ of the world's population aged 60 and above will be living in developing countries, with $14.2 \%$ in India. ${ }^{7}$ The reported incidence rates for $\mathrm{AD}$ have been lower in Asian countries than in the industrialized world. ${ }^{8}$ Another projection indicated that the number of people affected by dementia will double between 2020 (42 million) and 2040 (81million). ${ }^{5}$

There are currently about 18 million people worldwide with Alzheimer's diseases. In India the Alzheimer's cases are estimated to be 3.5 million, though majority of the problem remains hidden. ${ }^{9}$

Currently, the mainstay treatments for $\mathrm{AD}$ are the cholinesterase inhibitors, which increase the availability of acetylcholine at cholinergic synapses. Since the cholinesterase inhibitors confer only modest benefits, additional non-cholinergic therapies are currently under development for the treatment and/or prevention of AD. Drug research in the last decade has attempted to develop disease-modifying drugs hopefully able to delay the onset or counteract the progression of AD.

These include anti-amyloid strategies (e.g. immunization, aggregation inhibitors, secretase inhibitors), transition metal chelators (e.g. clioquinol), growth factors, hormones (e.g. estradiol), herbs (e.g. Ginkgo biloba), nonsteroidal anti-inflammatory drugs (NSAIDs, e.g. indomethacin), antioxidants, lipid-lowering agents, antihypertensives, selective phosphodiesterase inhibitors, vitamins (E, B12,
B6, folic acid) and agents that target neurotransmitter or neuropeptide alterations. Neurotransmitter receptor-based approaches include agents that modulate certain receptors (e.g. nicotinic, muscarinic, $\alpha$-amino-3-hydroxy-5-methyl4-isoxazole proprionic acid [AMPA], $\gamma$-aminobutyric acid [GABA], $N$-methyl-D-aspartate [NMDA]) and agents that increase the availability of neurotransmitters (e.g. noradrenergic reuptake inhibitors). ${ }^{10}$ Drugs targeting tauprotein include: modulators of tau phosphorylation, inhibitors of tau-phosphorylating kinases (e.g. glycogensynthase-kinase-3 $\beta$, cyclin-dependent kinase-5, p70-S6kinase) and compounds that prevent tau aggregation and misfolding. ${ }^{11}$

\section{METHODS}

Adult Swiss albino mice ( $\mathrm{n}=$ ninety six) of either sex weighing between 25- 35 grams were used for this study. Albino mice were divided into 4 sets each consisting of 4 groups with 6 animals in each group. Mice were housed in groups of six in polypropylene cages with wood shavings as bedding, under controlled conditions of light at room temperature $\left(25 \pm 2^{0} \mathrm{C}\right)$ and humidity of $55 \%$ under $12 \mathrm{~h}$ light-dark cycle.

All animals were allowed to acclimatize with laboratory conditions at least two weeks before starting the experiment and they were maintained under the same condition throughout the experiment. They were given food and water ad libitum. The experiments were performed as per the Committee for the Purpose of Control and Supervision on Experiments on Animals (CPCSEA) guidelines. The animals were subjected to experimentation between 0900-1600 hours in noise free atmosphere with ambient temperature $23-30^{\circ} \mathrm{C}$.

\section{Animals used}

- $\quad$ Species: Albino mice of Swiss strain.

- Age (months): Around 3-4 months.

- Gender: Both males and females.

- Weight: 25 -35 grams.

- Number to be used: Ninety six.

- Number of days each animal was housed: one month.

\section{Inclusion criteria}

- $\quad$ Swiss Albino mice weighing 25 to $35 \mathrm{~g}$ of either sex.

- $\quad$ Age 3-4 months.

- Animals acclimatized to experimental conditions for two weeks.

- Healthy mice with normal behavior and activity.

\section{Exclusion criteria}

- $\quad$ Mice weighing more than $35 \mathrm{~g}$ and less than $25 \mathrm{~g}$.

- Diseased mice.

- Pregnant mice 


\section{RESULTS}

Effect of different doses of Pioglitazone on learning and memory was tested using a passive avoidance paradigm. In this experiment the parameter Step down latency (SDL) was recorded for each animal in all the 4 test groups $(n=$ $6)$.

The duration of time taken by the mice to step down from an elevated platform to the shock zone is called step down latency. When compared to normal control group, the scopolamine treated group showed reduced step down latency on both acquisition day and retention day. The Pioglitazone treated groups showed statistically significant results in the SDL when compared to the control group ( $p<0.01$ on both days) and the scopolamine group $(\mathrm{p}<0.01$ on both days) on both acquisition day and retention day. There was no significant difference in SDL in standard and Pioglitazone treated groups on both acquisition day and retention day.

Table 1: Effect of Pioglitazone pre-treatment in scopolamine induced amnesia on passive avoidance paradigm: step down latency (SDL) on acquisition day (day 7) and retention day (day 8).

\begin{tabular}{|lllll|}
\hline Groups $(\mathbf{n}=\mathbf{6})$ & & Treatment & \multicolumn{2}{l|}{ Step down latency (SDL) Mean \pm SD (in seconds) } \\
\cline { 3 - 4 } & & Acquisition day & Retention day \\
\hline 1 & Control & Normal Saline & $36 \pm 4.98$ & $78.67 \pm 8.14$ \\
\hline 3 & Negative control & Scopolamine & $12.5 \pm 4.64$ & $28.17 \pm 4.4$ \\
\hline 4 & Standard & Piracetam & $66.17 \pm 4.71$ & $178.67 \pm 19.29$ \\
\hline
\end{tabular}

Data represented as Mean \pm SD $(n=6)$. Analyzed by One way ANOVA followed by post hoc Dunnett's multiple comparison test. $\mathrm{a}=$ Compared with control, $\mathrm{b}=$ Compared with negative control

Significant at $\mathrm{p}<0.05^{*}, \mathrm{p}<0.01 * *$ and $\mathrm{p}<0.001 * * *$

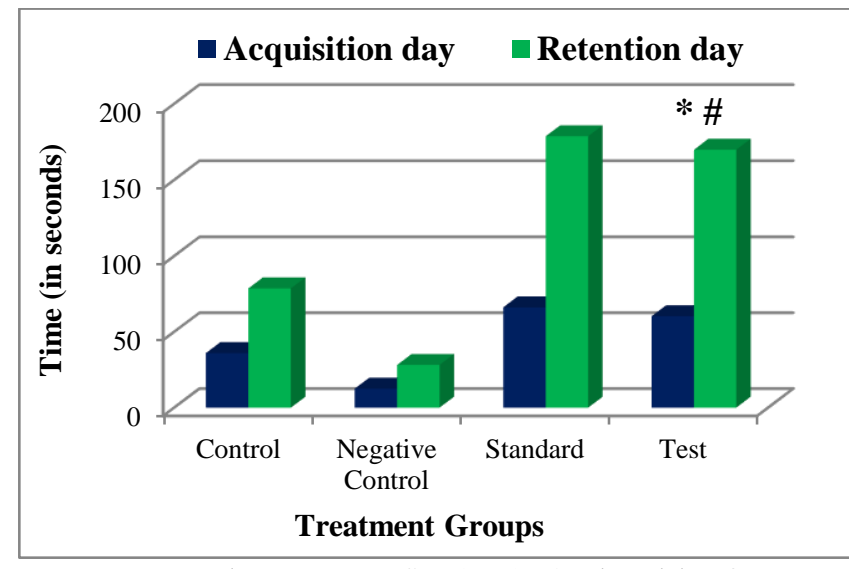

Data represented as Mean \pm SD $(n=6)$. Analyzed by Oneway ANOVA followed by post hoc Dunnett's multiple comparison test.

Significant at $\mathrm{p}<0.05$

Compared with control - *

Compared with negative control - \#

Figure 1: Effect of pioglitazone pretreatment in scopolamine induced amnesia on passive avoidance paradigm: step down latency (in sec) on acquisition day (day 7) and retention day (day 8).

\section{DISCUSSION}

The avoidance paradigm is based on Pavlovian fear conditioning. Pavlovian defensive reactions depend on a well characterized amygdalar substrate. Results from studies in animals and humans have strongly implicated amygdala as the primary orchestrator of the process of emotional memory and the site of neuroplasticity underlying memory storage of conditioned fear as well as an unconditioned one. ${ }^{12}$ The impairment of emotional event memory in $\mathrm{AD}$ is related to the intensity of damage of amygdala. ${ }^{13}$ Aversive conditioned stimuli can evoke a contrasting class of defensive behaviors, such as escape and avoidance responses, that allow the subject to influence or control the impact of environmental threats.

Table 2: Group wise comparison of step down latency on acquisition day (day 7) and retention day (day 8).

\begin{tabular}{|c|c|c|c|c|}
\hline \multirow[b]{2}{*}{$\begin{array}{l}\text { Groups } \\
\text { compared }\end{array}$} & \multicolumn{2}{|c|}{ Acquisition day } & \multicolumn{2}{|c|}{ Retention day } \\
\hline & $\begin{array}{l}\text { Mean } \\
\text { difference } \\
\text { (in seconds) }\end{array}$ & $\begin{array}{l}\text { P- } \\
\text { value* }\end{array}$ & $\begin{array}{l}\text { Mean } \\
\text { difference } \\
\text { (in seconds) }\end{array}$ & $\begin{array}{l}\text { P- } \\
\text { value }^{*}\end{array}$ \\
\hline $1-$ & -24.33 & $\begin{array}{l}0.001 \\
\mathrm{~S}\end{array}$ & -91.16 & $\begin{array}{l}0.001 \\
\mathrm{~S}\end{array}$ \\
\hline $2-$ & -47.83 & $0.001 \mathrm{~S}$ & -141.66 & $\begin{array}{l}0.001 \\
S\end{array}$ \\
\hline 3- & 5.84 & $\begin{array}{l}0.158 \\
\text { NS }\end{array}$ & 8.84 & $\begin{array}{l}0.497 \\
\mathrm{NS}\end{array}$ \\
\hline
\end{tabular}

$\mathrm{P}>0.005$ - not significant- NS

$\mathrm{P}<0.005$ - significant- $\mathrm{S}$

In the avoidance models, the amygdala is impaired through foot electric shocks (shuttle box model) which contribute to the impairment of memory. Active avoidance learning is a fundamental behavior phenomenon wherein it requires the subjects to behave contrary to their innate tendencies for preference of dark areas and avoidance of bright ones. The first stage of avoidance learning is escape behavior- 
the animal relocates to the adjoining compartment within a preset time in order to avoid the mild electric shock. The latency from stimuli onset to escape of the subject after the pre-training is related to the retention of memory task. ${ }^{14}$ However, in the present study we have used the passive avoidance paradigm- (step down) which is a classic model for the assessment of cognitive performance after brain lesions or pharmacological manipulation.

Passive avoidance response is widely used for the screening of drugs affecting learning and memory. In this model, the avoidance cage consists of a single box without any compartments that prevents inter-compartment transfers. Here, the rodent is trained to associate shock delivery with stepping off an elevated platform by inhibiting the normal exploratory behavior. A sequence of stimuli is presented during which the animal has the freedom to move onto a safe area within the compartment. Failure to transfer or the latency to move to a shock-free area indicates passive behavior which is used as a shortterm memory task. After 24 hours, the animals were tested again for retention of such learning. The parameter used here was step down latency (SDL).

Memory performance is positively correlated with the latency to transfer from the shock free zone (SDL); the better the recollection, the greater the latency.

The inhibitory avoidance test is a classic model behavioral test, with a strong aversive component, utilized for evaluating learning and memory in mice.

In step- down latency (SDL) using the passive avoidance paradigm, the scopolamine-treated mice showed significantly shorter latency times on both acquisition and retention days suggesting learning and memory impairment, when compared to the control group which is consistent with the study by Nakajima. ${ }^{15}$ The standard and the Pioglitazone groups significantly increased SDL, which is suggestive of improvement of cognition, when compared with the control group as reported by Kaur et al. ${ }^{16}$ Pioglitazone significantly improved the SDL compared to scopolamine-treated mice $(p<0.01)$. There was no significant difference in SDL in standard and Pioglitazone treated groups on both acquisition day and retention day suggesting that cognitive enhancing activity of Pioglitazone is similar to that of the standard drug Piracetam.

The antagonism of scopolamine induced amnesia by Pioglitazone on both learning and retention testing is comparable to that of standard drug Piracetam in the SDL parameter observed in passive avoidance step down paradigm.

The elevated plus maze is one of the many tests used to measure the anxiety (anxiolytic or anxiogenic) state in animals, however transfer latency (TL) i.e. the time elapsed between movements of the animal from an open arm to a closed arm will be markedly reduced if the animal had previously experienced entering from open arm to closed arms and this shortened transfer latency has been shown to be related with memory processes. Recent studies of several nootropics and amnestic agents on EPL made this model a widely accepted paradigm to study learning and memory process in rodents. In EPL, learning or acquisition can be considered as transfer latency on first-day trials and the retention or consolidation (memory) is examined $24 \mathrm{~h}$ later. ${ }^{17}$

In the present study the test drug Pioglitazone was compared with the control group, standard drug Piracetam and negative control scopolamine treated mice. TL was statistically significant in Pioglitazone treated group as compared with the normal control group indicating enhanced cognitive effect. In the present study, Scopolamine significantly prolonged TL in mice, indicating its amnesic effects which is consistent with the results reported by Itoh et al. ${ }^{18}$

The standard drug Piracetam reduced TL, whereas negative control scopolamine enhanced TL significantly, suggesting impairment in cognitive behavior with Scopolamine which was antagonized by piracetam. The Pioglitazone group showed significant reduction in TL compared to the scopolamine control on both days.

Though the cholinergic hypothesis implicated in geriatric memory disorders in very intriguing, cholinergic deficits cannot solely explain the age- associated deficits of cognitive functions. Excitotoxicity mediated by over activation of NMDARs has been implicated in a variety of neuropathological conditions in the CNS including AD.

The hippocampus is closely related to learning and memory, has a very high density of glutamate receptors, particularly the NMDA-type, which are significantly involved in neuronal plasticity. Glutamate is essential for the establishment of new neural networks, forming memory and learning through long-term potentiation (LTP) or long-term depression (LTD) of synaptic strength, which occurs upon activation of NMDARs. In AD patients, the glutamatergic transmission is severely affected by neurons in the cortex and hippocampus.

The continuous presence of glutamate induces constant activation of the NMDAR, and the ensuing massive influx of $\mathrm{Ca}^{2+}$ contributes to the excitotoxicity and may trigger a cascade of events leading to neuronal injury and death. ${ }^{19}$ Chronic depolarization of the membrane on vulnerable neurons, as observed in AD patients, is also accompanied by neuronal oxidative stress, mitochondrial damage, and inflammation, and the presence of $A \beta$ and possibly hyper phosphorylated- tau, which may eventually increase the sensitivity of the glutamatergic system and result in neuronal dysfunction and cell death.

The general hypothesis states that increased $\mathrm{Ca}^{2+}$ transients and $\mathrm{Ca}^{2+}$ - dependent processes during aging distort $\mathrm{Ca}^{2+}$ signaling and synaptic plasticity, leading to impaired 
neuronal communication, and finally to impaired cognition, memory formation and neurodegenerative changes.

The Scopolamine group significantly increased Transfer latency on both days compared to control group. The TL values of the Pioglitazone treated group showed statistical significance compared to the control $(\mathrm{p}<0.01$ and $<0.001)$ and scopolamine $(\mathrm{p}<0.01$ and $\mathrm{p}<0.0001)$ groups on both acquisition day and retention day. The TL on retention day was significantly shorter than on acquisition day in Pioglitazone treated group. However, the Pioglitazone treated group did not show statistical significance in TL, when compared to the standard group on both acquisition day and retention day.

\section{CONCLUSION}

The results from this study have demonstrated that PIO has reversed the pharmacologically induced amnesia by Scopolamine. Many mechanisms are postulated for the cognitive enhancing activity of PIO. Primarily it may act on the glutamatergic pathway in the brain where it ameliorates the $\mathrm{Ca}^{2+}$ mediated excitotoxicity by its antagonistic action on NMDAR and improves the memory function.

\section{Funding: No funding sources}

Conflict of interest: None declared

Ethical approval: The study was approved by the Institutional Ethics Committee

\section{REFERENCES}

1. Rubinsztein DC. The roles of intracellular proteindegradation pathways in neurodegeneration. Nature. 2006;443(7113):780-6.

2. Bredesen DE, Rao RV, Mehlen P. Cell death in the nervous system. Nature. 2006;443(7113):796-802.

3. Horton R. GBD 2010: understanding disease, injury and risk. The Lancet. 2012;380(9859):2053-4.

4. Dementia. Fact sheet $N^{\circ}$ 362. March 2015 [accessed 24 September 2015]. Available at: http://www.who.int/mediacentre/factsheets/fs362/en/

5. Ferri CP, Prince M, Brayne C, Brodaty H, Fratiglioni L, Ganguli M, Hall K, Hasegawa K, Hendrie H, Huang Y, Jorm A. Global prevalence of dementia: a Delphi consensus study. The lancet. 2006 Jan 6;366(9503):2112-7.

6. Forette F, Boller F. Hypertension and the risk of dementia in the elderly. The American $\mathrm{J}$ of Med. 1991;90(3).

7. World Health Organization. Population Ageing: A Public Health Challenge. Fact Sheet No. 135. Geneva: World Health Organization; 1998.
8. Mathuranath PS, George A, Ranjith N, Justus S, Kumar MS, Menon R, et al. Incidence of Alzheimer's disease in India: A 10 years follow-up study. Neurology India. 2012 Nov;60(6):625.

9. Gaugler j, Bryan J, Tricia J. Alzheimer's Association. 2013 Alzheimer's disease Facts and Figures. Alzheimer's \& Dementia. 2013;8(2):14-23.

10. Murali DP. Non-Cholinergic Strategies for Treating and Preventing Alzheimer's Disease. CNS Drugs. 2002;16(12):811-24.

11. Mangialasche F, Solomon A, Winblad B, Mecocci P, Kivipelto M. Alzheimer's disease: clinical trials and drug development. Lancet Neurol. 2010;9(7):702-16.

12. McGaugh JL. Amygdala: role in modulation of memory storage. In the Amygdala: A Functional Analysis; 2 $2^{\text {nd }}$ Ed. (Aggleton, J.P., ed.), Oxford University Press; 2000:391-423.

13. Mori E, Ikeda M, Hirono N, Kitagaki H, Imamura T. Amygdalar volume and emotional memory in Alzheimer's disease. Am J Psychiatry. 1999;156(2):216-22.

14. Reddy DS. Assessment of nootropic and amnestic activity of centrally acting agents. Indian J Pharmacol. 1997;29:208-21.

15. Nakajima M, Inui A, Miura M, Hirosue Y, Okita M. Negative and positive effects of intracerebroventricular scopolamine on memory in mice undergoing passive avoidance and escape tests. Brain Res Bull. 1994;34(4):375-80.

16. Kaur B, Singh N, Jaggi AS. Exploring mechanism of pioglitazone-induced memory restorative effect in experimental dementia. Fundamental \& clinical pharmacology. 2009 Oct 1;23(5):557-66.

17. Achliya G, Barabde U, Wadodkar S, Dorle A. Studies on the effects of Bramhi ghirta, an polyherbal formulation on learning and memory paradigms in experimental animals. Ind $\mathbf{J}$ Pharmacol. 2004;36(3):159-62.

18. Itoh $J$, Nabeshima $T$, Kameyama $T$. Utility of an elevated plus-maze for dissociation of amnesic and behavioral effects of drugs in mice. Eur J Pharmacol. 1991;194(1):71-6.

19. Bezprozvanny I, Hiesinger PR. The synaptic maintenance problem: membrane recycling, $\mathrm{Ca}^{2+}$ homeostasis and late onset degeneration. Mol Neurodegener. 2013;8:23.

Cite this article as: Shruthi SL, Kalabharathi HL, Amoghimath S. Evaluation of cognition enhancing activity of pioglitazone in albino mice. Int J Basic Clin Pharmacol 2017;6:2733-7. 